\title{
Protective effects of mito-TEMPO against doxorubicin cardiotoxicity in mice
}

\author{
Viviane Costa Junqueira Rocha ${ }^{1} \cdot$ Luciana Souza de Aragão França $^{1} \cdot$ \\ Cintia Figueiredo de Araújo ${ }^{1} \cdot$ Ayling Martins $\mathrm{Ng}^{1} \cdot$ Candace Machado de Andrade $^{1}$. \\ André Cronemberger Andrade ${ }^{2} \cdot$ Emanuelle de Souza Santos $^{1}$. \\ Mariana da Cruz Borges-Silva ${ }^{1} \cdot$ Simone Garcia Macambira $^{1}$. \\ Alberto Augusto Noronha-Dutra ${ }^{1,3} \cdot$ Lain Carlos Pontes-de-Carvalho ${ }^{1}$
}

Received: 7 October 2015 / Accepted: 11 December 2015 / Published online: 28 December 2015

(C) Springer-Verlag Berlin Heidelberg 2015

\begin{abstract}
Purpose Doxorubicin (DOX) is a chemotherapeutic that is widely used for the treatment of many human tumors. However, the development of cardiotoxicity has limited its use. The aim of the present study was to evaluate the possible efficacy of mito-TEMPO (mito-T) as a protective agent against DOX-induced cardiotoxicity in mice.

Methods C57BL/6 mice were treated twice with mito$\mathrm{T}$ at low $(5 \mathrm{mg} / \mathrm{kg}$ body weight $)$ or high $(20 \mathrm{mg} / \mathrm{kg}$ body weight) dose and once with DOX ( $24 \mathrm{mg} / \mathrm{kg}$ body weight) or saline $(0.1 \mathrm{~mL} / 20 \mathrm{~g}$ body weight $)$ by means of intraperitoneal injections. The levels of malondialdehyde (MLDA), a marker of lipid peroxidation, and serum levels of creatine kinase were evaluated $48 \mathrm{~h}$ after the injection of DOX.

Results DOX induced lipid peroxidation in heart mitochondria $(p<0.001)$, and DOX-treated mice receiving mito-T at low dose had levels of MLDA significantly lower than the mice that received only DOX $(p<0.01)$. Furthermore, administration of mito-T alone did not cause any significant changes from control values. Additionally, DOX-treated mice treated with mito-T at high dose showed decrease in serum levels of total CK compared to mice treated with DOX alone $(p<0.05)$.
\end{abstract}

Lain Carlos Pontes-de-Carvalho

lain.carvalho@gmail.com

1 Gonçallo Moniz Research Center, Fundação Oswaldo Cruz, Rua Waldemar Falcão 121, Salvador, BA 40296-710, Brazil

2 Laboratório de Imunologia Celular e Bioquímica de Fungos e Protozoários, Universidade Federal de São Paulo, São Paulo, SP, Brazil

3 University College London, Gower Street, London, UK
Conclusion Our results indicate that mito-T protects mice against DOX-induced cardiotoxicity.

Keywords Doxorubicin - Cardiotoxicity · Mitochondria · Mito-TEMPO

\section{Introduction}

Doxorubicin (DOX) is an anthracycline chemotherapeutic that is widely used for the treatment of many human tumors since the late 1960s [1]. Its discovery represented one of the great advancements in the fight against cancer; however, the development of adverse drug reactions, in particular cardiotoxicity, has limited its use [2].

Although DOX-induced cardiac toxicity appears to be multifactorial, the most thoroughly investigated hypothesis has been the formation of reactive oxygen species (ROS) and there is evidence pointing to cardiac mitochondria as primary targets of the toxicity of DOX [3]. The quinone moiety of DOX may form semiquinone radicals by oneelectron reduction. This semiquinone, in the presence of molecular oxygen, results in the formation of superoxide anion and other ROS. Furthermore, it has been reported that DOX shows a high affinity for cardiolipin, a phospholipid in the inner mitochondrial membrane, which results in their accumulation inside cardiac cells and oxidative damage [4].

The possibility that cardiac dysfunction may lead to congestive heart failure stimulates the development of strategies to prevent or reduce DOX cardiotoxicity in the clinic, and the use of antioxidants could be an important strategy [5].

The mito-TEMPO (mito-T) is a nitroxide conjugated with a triphenylphosphonium (TPP) moiety that is 


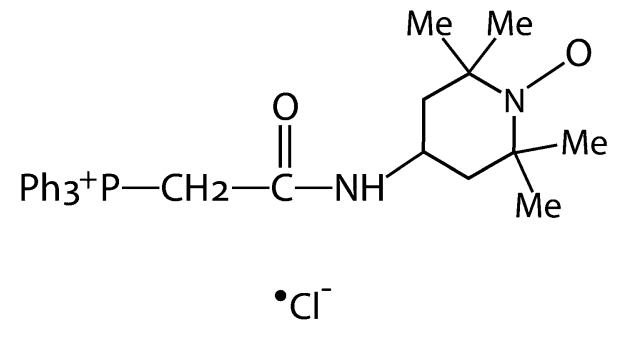

Fig. 1 Chemical structure of mito-TEMPO $\left(\mathrm{C}_{29} \mathrm{H}_{35} \mathrm{~N}_{2} \mathrm{O}_{2} \mathrm{P} \cdot \mathrm{Cl}\right)$

mitochondria-targeted and have been used as antioxidant. Since nitroxides are known to be superoxide dismutase (SOD)-mimetics, mito-T may act as a mitochondrial superoxide scavenger and to protect mitochondria from the DOX-induced oxidative damage [6]. Recent studies documented that mito- $\mathrm{T}$ decreased mitochondrial superoxide levels and increased survival rate from septic mice [7], attenuated TNF- $\alpha$-induced mitochondrial ROS and apoptosis in H9c2 cells [8] and blocked mitochondrial ROS generation and reduced DOX-induced platelet apoptosis [9].

Thus, as DOX produces significant amounts of ROS and has affinity for mitochondria, inducing mitochondrial and cardiac oxidative damage, the aim of the present study was to evaluate the possible efficacy of mito-T as a protective agent against DOX-induced cardiotoxicity in mice.

\section{Materials and methods}

\section{Animals and ethical approval}

Six- to ten-week-old female C57BL/6 mice were obtained and maintained at the animal facilities at the Gonçalo Moniz Research Center-FIOCRUZ (Salvador, Bahia, Brazil). The animals received balanced feed and water ad libitum.

The current work was carried out in accordance with the Brazilian Federal Law on Animal Experimentation (Law 11794) (http://www.planalto.gov.br/ccivil_03/_ato20072010/2008/lei/111794.htm). The protocol was approved by the Ethics Committee for the Use of Animals in Research (CPqGM-FIOCRUZ, CEUA, license number 019/2012).

\section{Design of the work}

Seven to eight mice from each group were treated twice with mito-T (Fig. 1; Sigma-Aldrich Chemical Co, St. Louis, MO, USA) at low ( $5 \mathrm{mg} / \mathrm{kg}$ body weight $)$ or high (20 mg/kg body weight) dose and once with DOX (Glenmark Farmacêutica Ltda, São Paulo, SP, Brazil; 24 mg/ $\mathrm{kg}$ body weight) or saline (Farmace, Barbalha, CE, Brazil; $0.1 \mathrm{~mL} / 20 \mathrm{~g}$ body weight) by means of intraperitoneal injections, as described below. The mice were euthanized $48 \mathrm{~h}$ after DOX by carbon dioxide inhalation.

Low dose of mito-T:

Group 1-Saline and saline;

Group 2-mito-T (5 mg/kg) twice, 48 and $24 \mathrm{~h}$ before

saline;

Group 3-mito-T ( $5 \mathrm{mg} / \mathrm{kg})$ twice, 48 and $24 \mathrm{~h}$ before

DOX $(24 \mathrm{mg} / \mathrm{kg})$;

Group 4-DOX (24 mg/kg).

High dose of mito-T:

Group 1-Saline and saline;

Group 2-mito-T (20 mg/kg) twice, 24 and $1 \mathrm{~h}$ before saline;

Group 3-mito-T (20 mg/kg) twice, 24 and $1 \mathrm{~h}$ before

DOX $(24 \mathrm{mg} / \mathrm{kg})$;

Group 4-DOX (24 mg/kg).

\section{Determination of lipid peroxidation in heart mitochondria}

Hearts of animals treated with low dose of mito-T were collected and mitochondria obtained as described by Fernández-Vizarra et al. [10]. Heart mitochondria lipid peroxidation was quantified by measuring the thiobarbituric acid reactive substance, malondialdehyde (MLDA). An aliquot of heart mitochondria suspension $(100 \mu \mathrm{L})$ was incubated with $185 \mu \mathrm{L}$ of a solution containing acetic acid (SigmaAldrich) $50 \%$ in water, $1.3 \%$ thiobarbituric acid (Merck KGaA, Darmstadt, HES, Germany) and phosphate buffer solution, $\mathrm{pH}$ 7.2, in proportion 1:1.5:1.2, respectively, for $30 \mathrm{~min}$ at $90{ }^{\circ} \mathrm{C}$. After that, the samples were centrifuged (9000g for $10 \mathrm{~min}$ at $4{ }^{\circ} \mathrm{C}$ ) and the supernatants collected for measurement of absorbance at $532 \mathrm{~nm}$ using a multiplate reader (SpectraMax, Molecular Devices, Sunnyvale, CA, USA). Malondialdehyde (Sigma-Aldrich) was used as a standard.

\section{Quantification of serum creatine kinase}

Blood samples of animals treated with high dose of mito-T were taken and centrifuged at $1200 \mathrm{~g}$ for $20 \mathrm{~min}$ at $4{ }^{\circ} \mathrm{C}$. Serum was collected and quantified for total creatine kinase (CK) and CK-MB using diagnostic kits (Gold Analisa Diagnóstica Ltda, Belo Horizonte, MG, Brazil), following the manufacturer's recommendations.

\section{Statistical analysis}

The normality of the data was assessed by the D'Agostino and Pearson normality test. Statistical differences were 


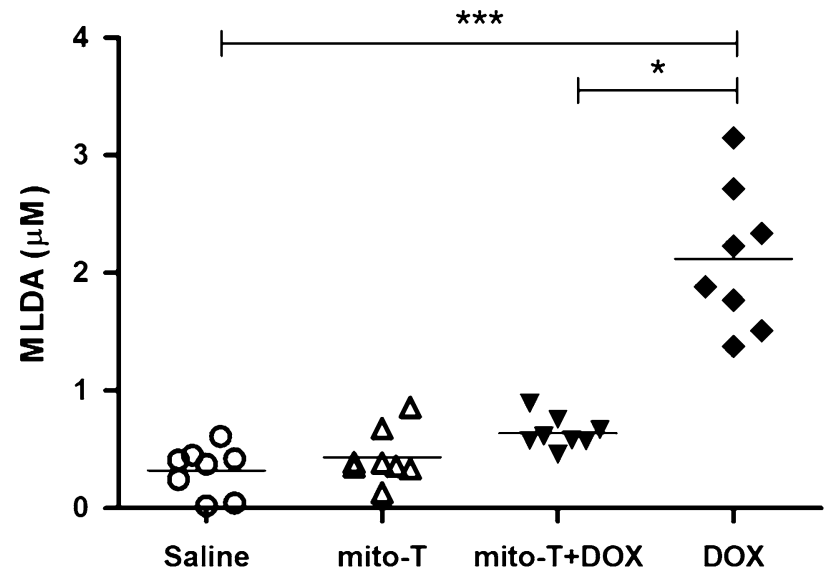

Fig. 2 Lipid peroxidation in heart mitochondria of mice treated with two mito-TEMPO (mito-T; $5 \mathrm{mg} / \mathrm{kg}$ body weight) doses and/or once with doxorubicin (DOX; $24 \mathrm{mg} / \mathrm{kg}$ body weight). Mice were treated intraperitoneally with mito-T, 48 and $24 \mathrm{~h}$ before administration of DOX. Heart mitochondria lipid peroxidation was quantified by measuring the malondialdehyde (MLDA). Negative control mice received only saline. Each symbol represents the result obtained from a single animal. Horizontal lines represent the median values for groups of eight animals. Comparisons among groups were performed by Dunn's multiple comparison test. $* p<0.05 ; * * * p<0.001$

analyzed using Kruskal-Wallis test followed by Dunn's test. Statistical significance was accepted at $p \leq 0.05$. All analysis was performed using the GraphPad Prism 5.0 Software (San Diego, CA, USA).

\section{Results}

DOX induced lipid peroxidation in heart mitochondria $(p<0.001)$, and DOX-treated mice receiving mito-T at low dose showed level of MLDA significantly lower compared

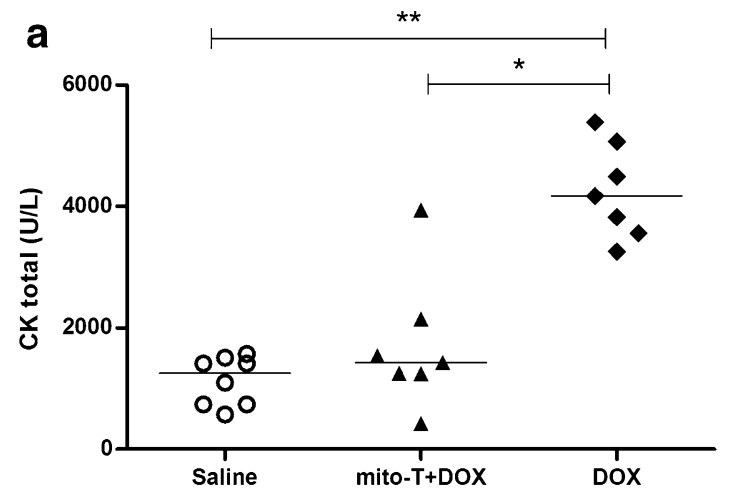

Fig. 3 Serum levels of creatine kinase (CK) total (a) and CK-MB (b) in heart of mice treated with two mito-TEMPO (mito-T; $20 \mathrm{mg} /$ $\mathrm{kg}$ body weight) doses and/or once with doxorubicin (DOX; $24 \mathrm{mg}$ / $\mathrm{kg}$ body weight). Mice were treated intraperitoneally with mito-T, 24 and $1 \mathrm{~h}$ before administration of DOX. Each symbol represents the to DOX group $(p<0.01)$. Furthermore, administration of mito-T alone did not cause any significant change from control values (Fig. 2).

Additionally, the mice treated with mito-T at high dose showed decrease in serum levels of total CK compared to DOX alone $(p<0.05)$. Although not significantly, the levels of CK-MB were reduced compared to DOX-treated group (Fig. 3). No differences were seen between mito-T-injected and mito-T-untreated mice in the low-dose mito-T experiment (data not shown).

\section{Discussion}

The search to new substances and therapeutic strategies to attenuate chemotherapy toxicity continues. As the cardiac susceptibility to DOX-induced oxidative stress has been associated with the high oxidative metabolism of the heart and its lower level of antioxidant enzymes and with the affinity of DOX to cardiolipin [11, 12], in the present investigation it was evaluated whether mito-T pretreatment could produce cardioprotective effects in mice treated with DOX.

Pretreatment of animals with mito-T $(5 \mathrm{mg} / \mathrm{kg}, 48$ and $24 \mathrm{~h}$ prior to DOX) significantly reduced the lipid peroxidation of heart mitochondria $(p<0.01)$ indicating targeting of the antioxidant to the mitochondria, as referred by Dikalova et al. [6]. Lipid peroxidation is considered a good cardiotoxicity indicator for DOX [4]; therefore, their reduction indicates that mito-T probably acts as an antioxidant that could prevent the mitochondrial disfunction DOX induced and its consequently cardiotoxicity.

Increase in CK serum level after DOX administration is referred as a consequence of heart injury [13]. Pretreatment with mito- $\mathrm{T}(20 \mathrm{mg} / \mathrm{kg})$ twice, 24 and $1 \mathrm{~h}$ before

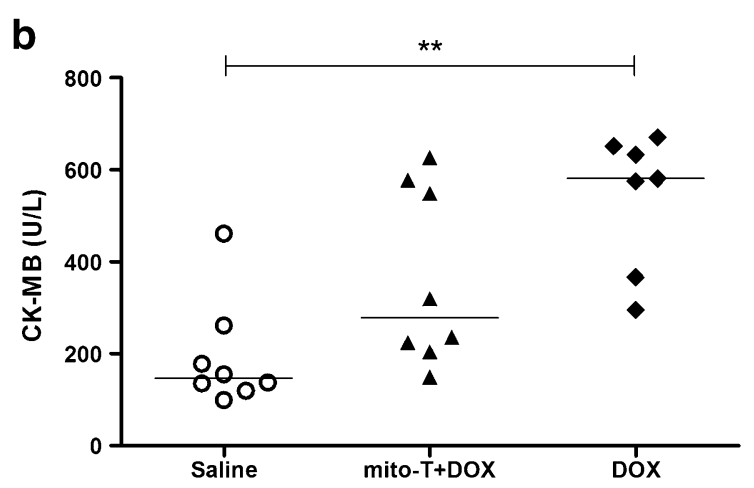

result obtained from a single animal. Horizontal lines represent the median values for groups of seven or eight animals. Comparisons among groups were performed by Dunn's multiple comparison test. $* p<0.05 ; * * p<0.01$ 
DOX treatment, inhibited the increase in CK total serum level $(p<0.05)$ induced by DOX. No significant differences were observed in CK-MB serum levels, however, probably because of great variation within groups. In work evaluating different doses of mito-T, Patil et al. [7] suggest that accumulation of higher dose of mito-T within the mitochondria could depolarize membrane and impair their protector effect. Thus, the therapeutic effects of this antioxidant at high dose should be better investigated.

\section{Conclusion}

Our data suggest that mito-T at a relatively high dose protects mice against DOX-induced cardiotoxicity; however, as the precise mechanism of the antioxidant activity of mito-T remains unclear, further investigations are required to confirm this assumption.

Acknowledgments This work was financially supported by Fundação de Amparo a Pesquisa do Estado da Bahia-FAPESB (www.fapesb.ba.gov.br), State Government of Bahia, Brazil. VCJR received a scholarship from Fundação Oswaldo Cruz, Brazil. LCPC have productivity scholarship from Conselho Nacional de Desenvolvimento Científico e Tecnológico- $\mathrm{CNPq}$ (www.cnpq.br), Ministry of Science and Technology, Brazil.

\section{Compliance with ethical standards}

Conflict of interest The authors have declared that no competing interests exist.

\section{References}

1. Arcamone F, Franceschi G, Penco S, Selva A (1969) Adriamycin (14-hydroxydaunomycin), a novel antitumor antibiotic. Tetrahedron Lett 10:1007-1010. doi:10.1016/S0040-4039(01)97723-8

2. Lefrak EA, Piťha J, Rosenheim S, Gottlieb JA (1973) A clinicopathologic analysis of adriamycin cardiotoxicity. Cancer 32:302314. doi:10.1002/1097-0142(197308)32:2<302:AID-CNCR282 $0320205>3.0 . \mathrm{CO} ; 2-2$
3. Li B, Kim D, Yadav R et al (2015) Sulforaphane prevents doxorubicin-induced oxidative stress and cell death in rat $\mathrm{H} 9 \mathrm{c} 2$ cells. Int J Mol Med 36:53-64. doi:10.3892/ijmm.2015.2199

4. Costa VM, Carvalho F, Duarte JA et al (2013) The heart as a target for xenobiotic toxicity: the cardiac susceptibility to oxidative stress. Chem Res Toxicol 26:1285-1311. doi:10.1021/tx400130v

5. Granados-Principal S, Quiles JL, Ramirez-Tortosa CL et al (2010) New advances in molecular mechanisms and the prevention of adriamycin toxicity by antioxidant nutrients. Food Chem Toxicol 48:1425-1438. doi:10.1016/j.fct.2010.04.007

6. Dikalova AE, Bikineyeva AT, Budzyn K et al (2010) Therapeutic targeting of mitochondrial superoxide in hypertension. Circ Res 107:106-116. doi:10.1161/CIRCRESAHA.109.214601

7. Patil NK, Parajuli N, MacMillan-Crow LA, Mayeux PR (2014) Inactivation of renal mitochondrial respiratory complexes and manganese superoxide dismutase during sepsis: mitochondriatargeted antioxidant mitigates injury. Am J Physiol Ren Physiol 306:F734-F743. doi:10.1152/ajprenal.00643.2013

8. Pan S, Wang N, Bisetto S et al (2015) Downregulation of adenine nucleotide translocator 1 exacerbates tumor necrosis factor$\alpha$-mediated cardiac inflammatory responses. Am J Physiol Heart Circ Physiol 308:H39-H48. doi:10.1152/ajpheart.00330.2014

9. Wang Z, Wang J, Xie R et al (2015) Mitochondria-derived reactive oxygen species play an important role in doxorubicininduced platelet apoptosis. Int J Mol Sci 16:11087-11100. doi:10.3390/ijms 160511087

10. Fernández-Vizarra E, Ferrín G, Pérez-Martos A et al (2010) Isolation of mitochondria for biogenetical studies: an update. Mitochondrion 10:253-262. doi:10.1016/j.mito.2009.12.148

11. Abdella BR, Fisher J (1985) A chemical perspective on the anthracycline antitumor antibiotics. Environ Health Perspect 64:4-18. doi:10.1289/ehp.85644

12. Octavia Y, Tocchetti CG, Gabrielson KL et al (2012) Doxorubicin-induced cardiomyopathy: from molecular mechanisms to therapeutic strategies. J Mol Cell Cardiol 52:1213-1225. doi:10.1016/j.yjmcc.2012.03.006

13. Hassan MH, El-Beshbishy HA, Aly H et al (2014) Modulatory effects of meloxicam on cardiotoxicity and antitumor activity of doxorubicin in mice. Cancer Chemother Pharmacol 74:559-569. doi:10.1007/s00280-014-2544-3 